\title{
Moderation of Enterprise Social Networks - A Literature Review from a Corporate Perspective
}

\author{
Ferry Nolte \\ Leibniz Universität Hannover \\ nolte@iwi.uni-hannover.de
}

\author{
Nadine Guhr \\ Leibniz Universität Hannover \\ guhr@iwi.uni-hannover.de
}

\author{
Michael H. Breitner \\ Leibniz Universität Hannover \\ breitner@iwi.uni-hannover.de
}

\begin{abstract}
The implementation of internal social collaboration technologies confronts corporations with new challenges. Former unidirectional information flows become multidirectional and usercontent driven networks. Prior research describes the successful implementation as a challenging management task with employees' usage at the center of attention. Consequently, corporations need to select a moderation style to encourage the usage. The degree of corporate engagement might have repercussions on the contribution behavior. We conduct a structured literature review to identify pre-existing IS contributions to the moderation phenomenon in social media tools, which help to explain on how to moderate these communication platforms in the enterprise context. We reviewed over 150 articles on the subject and assessed 31 articles in depth on the degree of corporate engagement and user content encouragement. We analyze the identified literature for gaps in understanding the phenomenon and provide a first assessment of three different moderation approaches and give implication for future research.
\end{abstract}

\section{Introduction}

Enterprise social networks (ESN) is still a young field within the information systems (IS) research. The dynamic nature of this research field calls for a shifting focus to address the plethora issues, both from the perspective of research as well as from practice. Organizations nowadays are heavily investing into ESN services to facilitate a better exchange, wider reach and easier access to organizational information $[3,39,45]$. As a consequence of such high organizational attention, this young and evolving IS research field is recognized as an important part of the whole social media research stream [35]. Those web based social media technologies - micro-/blogs, social networking sites, wikis, communities to name a few $[17,36,67]$ - are common in user features of profiles, relational connections and sharing/exploring [8]. ESN services can be separated in intra-organizational platforms restricted to an employee's audience or in the use of social media platforms for external party interaction [59]. The first named intra-organizational context challenges enterprise settings fundamentally. Via ESN services intra-organizational knowledge and information flows become visible throughout the whole corporation to enable employee driven communication, collaboration, innovation and knowledge sharing [22,43]. The visibility of information and relation ties is achieved with the open display of user generated content (UGC) within the ESN [45]. Here the UGC plays a vital role for organizations, as the open content display can be beneficial for enterprises to bridge structural holes in terms of employee innovation [22] or diminishes the unwanted information brokerage of specific individuals' in the organization [60]. Further, the visibility lets employees explore UGC actively by editing and distributing or passively through consuming content $[41,67]$. This transparent bottom up or crowd approach might interfere or even contrast with current organizational structures and work practices [37].

The current body of knowledge (BoK) in regard to the intra-organizational deployment of ESN is driven by the discussion of the support or substitution of internal communication, collaboration and knowledge sharing practices $[31,45,67]$. Here the effect on employee performance of ESN use [1,28,41] and factors influencing employees motivation to engage or reject such platforms $[10,12,13,42,52,62,69]$ are growing fields. Since, these new collaborations tools are impacting the organizational relation und information association, they also interfere with hierarchy and power structures [6,67]. This very interesting research topic has not been getting the main interest, although, it was being mentioned and acknowledged by the ESN researchers. As prior research was mainly focused on the user perspective, we try to pick up this discussion from a corporate perspective with the following question: 
RQ: How can organizations moderate the social exchanges in ESN and is a corporate moderation necessary?

The paper is structured as follows: In the first section the research design and methodology are described. After presenting the analysis procedure, we report the results. We highlight with this systematic literature review what prior research gave for implications on how those platforms can be moderated. The identified moderation concepts and implications are categorized by the degree of corporate engagement. Following the discussion, if such interference by a guiding corporate hand is influencing the UGC creation and if it is really necessary when considering the user-centric ESN design, we give implications for research and practice. We conclude by pointing out limitations and giving an outlook for future research.

\section{Methodology}

Consistent with [71], we performed a rigorous and structured literature review of publications to identifying, analyzing, and conceptualizing relevant research literature pertaining to ESN moderation. The rationale to use a literature review method is to structure the current BoK in order to highlight what prior research has already uncovered and to conceptualize new opportunities for extending the evolving ESN research field. According to [20] we outlined a four phase process for this research paper: (1) problem formulation and database collection, (2) initial screening, (3) clustering and (4) in-depth evaluation, analysis and presentation of the data to synthesize and extend the current BoK. In doing so, we were able to take advantage of the suggestions and possible pitfalls associated with each step of this literature review [40]. The initial phase (1) contains the examination of authoritative sources to determine a problem specification and formulation taking the proposed focus and scope into account [18]. For creating an appropriate pool of research papers, [71] argue that researchers should employ a high quality selection of papers. Due to the very young research field, we did expand our literature database, from mainly top ranked IS publication $[49,56]$ to other journals and conferences that are not highly rated. This decision was made to include innovative ideas, often presented in conferences, which are building the starting point for discussions [65] or in lower ranked journals [48]. The conferences included the latest social media track proceedings at ICIS, as the leading high quality conference in IS research [11], ECIS, to provide the newest exchanges of the European researches community and HICSS, to account for the
Anglo-American counterpart of the ongoing ESN discussions. To ensure reliability which refers to the replicability of the search process [9], the literature search process was documented comprehensively. Validity is based on the selected databases, covered period, keywords used and the application of a forward and backward search [9]. To fulfill the requirement for validity, we searched through different databases: AISeL (136 hits), ScienceDirect (67 hits), IEEEXplore (47 hits), JSTOR (3 hits), SpringerLink (23 hits), ACM (21), Wiley (101 hits), Emerald (26 hits) and InformsOnline (194 hits). From there a forward and backward search was conducted. Both were performed manually. To account for different abstraction levels occurring in literature, we used different search terms (e.g. "enterprise social media", "enterprise social network", "enterprise social software", and "enterprise social networking"). To select relevant publications in the considered research field, inclusion and exclusion criteria were defined. First, only literature in English language and with a strong focus on ESN was considered. Second, non-academic publications (such as white papers) and those that did not specifically deal with ESN (e.g. public use) were excluded. As the research field is quite young [35] no past time interval restriction was applied and the upper limit was set for the latest HICSS'16 proceedings. Upon completion of the research process, the period was ten years (from 2006 to 2016). In Phase 2, the databases were searched to determine whether a publication contained at least one of the search terms in the title, abstract or keywords. In total, 618 publications were identified. If the field of search (i.e. title, abstract or keywords) could not be specified in the search query, a full-text search was conducted. We therefore downloaded and checked these papers for relevance again. In most cases, papers that we omitted either did not yield any insights with respect to our research object or used the keyword in a different manner. After application of the before mentioned exclusion criteria, the final number of 157 papers results. In Phase 3, we tagged each paper with keywords indicating its major topic. Afterwards, these papers were clustered by topics. This led to the final typology (see Table 1) of 16 topics related to ESN moderation cluster. The major topics they addressed were, e.g. "power", "top-management commitment", "governance", "feedback" and "corporate communication". All of them were then consolidated to the "moderation" category and are subject to an indepth analysis. The topics assigned are all related to the corporate interference. E.g. the topics "power" addressed content flow barriers related with corporate hierarchies. Whereas "content overflow" topics relate to content prioritization difficulties faced by corporations in promoting the usefulness of such tools. 
These diverse topics will provide distinct angles of issues met by companies in regard to ESN moderation.

\section{Table 1 - Moderation topic cluster}

\begin{tabular}{|cll|}
\hline Code & Topic & Cluster \\
\hline A & Empowerment & Moderation \\
B & Power & Moderation \\
C & Policies & Moderation \\
D & Corporate communication / business objective & Moderation \\
E & Content quality & Moderation \\
F & Content overflow & Moderation \\
G & Corporate knowledge diffusion & Moderation \\
H & Knowledge protection / ownership & Moderation \\
I & Control & Moderation \\
J & Hierarchy & Moderation \\
K & Governance & Moderation \\
L & Media synchronity / ambidexterity & Moderation \\
M & Leadership / top-management commitment & Moderation \\
N & Participation & Moderation \\
O & Moderation & Moderation \\
P & Feedback/employee voice & Moderation \\
\hline & &
\end{tabular}

Table 2 - Overview of literature clustering

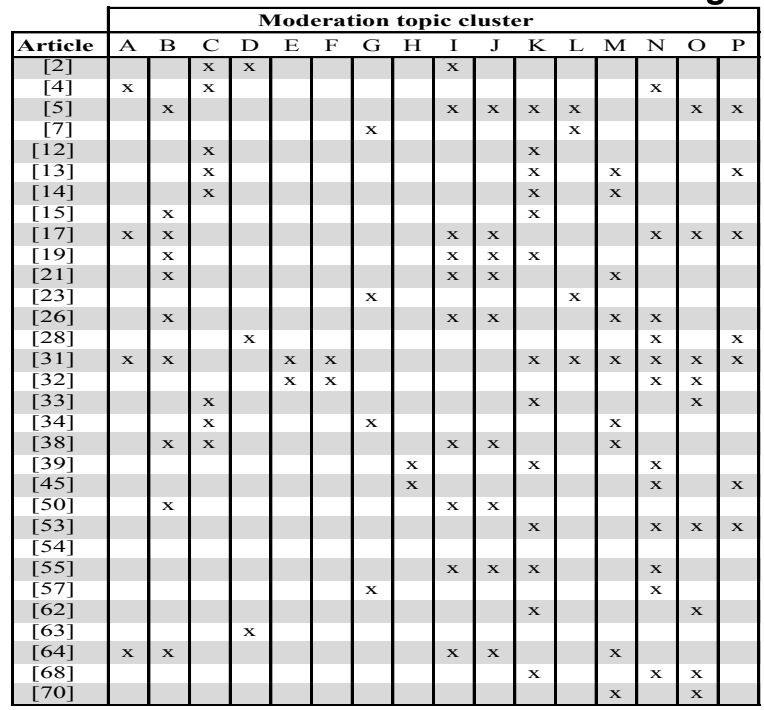

The final phase 4 include activities such as connecting, comparing and explaining [46]. We analyzed both the initially identified papers and the papers derived from the additional clustering process. Amounting up to 53 articles were than reviewed in depth, on their implications from a corporate perspective, which led to further eliminations from the publication base. In the end 31 articles are considered to be relevant for the discussion of the research question. Table 2 shows the final overview of the articles and their topic distribution within the derived moderation cluster, highlighting the diverse topics addressed within the articles.

\section{Research and findings}

The loss of control over the information flow or shift in power relation is concerning several researches in their discussion parts $[5,6,17,19,51,66]$. One could think that enterprises will be very cautious with implementing those tools but "executives and managers attempt to leverage the power of the informal information economies of their companies" $[38$, p. 747.]. Thus, the question on how to encourage this informal information networks to produce UGC is challenging. Considering on the one hand the loss of control and on the other hand the need for UGC is a contradicting goal conflict [5]. Since, the ESN is in an organizational setting the use might be mandatorily, promoted or voluntarily organized for employees [19]. This leads ultimately to a scenario in which the enterprise either controls and supervises the ESN content flow or leaves the organization to the employee crowd. Based on this diametric understanding a broad area in-between leaves room for further interpretation.

Table 3 - Overview of article grouping

\begin{tabular}{|c|c|c|c|}
\hline Article & $\begin{array}{c}\text { Corporate } \\
\text { supervisio } \\
n\end{array}$ & $\begin{array}{l}\text { Corporate } \\
\text { guidance }\end{array}$ & $\begin{array}{c}\text { Employee } \\
\text { self- } \\
\text { organizati }\end{array}$ \\
\hline [2] & $\mathrm{x}$ & & \\
\hline [4] & & & $\mathrm{x}$ \\
\hline [5] & $(\mathrm{x})$ & $\mathrm{x}$ & \\
\hline \multicolumn{4}{|l|}{ [7] } \\
\hline [12] & (x) & $\mathrm{x}$ & \\
\hline [13] & & $\mathrm{x}$ & \\
\hline [14] & & $\mathrm{x}$ & \\
\hline [15] & & & $\mathrm{x}$ \\
\hline [17] & & $\mathrm{x}$ & \\
\hline [19] & & $\mathrm{x}$ & \\
\hline [21] & & $\mathrm{x}$ & \\
\hline [23] & & $\mathrm{x}$ & \\
\hline [26] & & $\mathrm{x}$ & \\
\hline [28] & & $\mathrm{x}$ & \\
\hline [3 11$]$ & (x) & $\mathrm{x}$ & (x) \\
\hline [32] & $\mathrm{x}$ & & \\
\hline [33] & & $\mathrm{x}$ & \\
\hline [34] & & $\mathrm{x}$ & \\
\hline [38] & & $\mathrm{x}$ & \\
\hline [39] & & & $\mathrm{x}$ \\
\hline [45] & & & (x) \\
\hline [50] & $\mathrm{x}$ & & \\
\hline [53] & & $\mathrm{x}$ & \\
\hline [54] & & (x) & \\
\hline [55] & & (x) & (x) \\
\hline [57] & & (x) & $(\mathrm{x})$ \\
\hline [62] & & $\mathrm{x}$ & \\
\hline [63] & & $(\mathrm{x})$ & $(\mathrm{x})$ \\
\hline [64] & & $(\mathrm{x})$ & $(\mathrm{x})$ \\
\hline [68] & $\mathrm{x}$ & & \\
\hline [70] & & $\mathrm{x}$ & \\
\hline (x) not soley in favor & 3 & 5 & 6 \\
\hline $\mathrm{x}$ in favor & 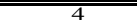 & 17 & 3 \\
\hline
\end{tabular}

Table 3 gives a brief overview about the arrangement of the reviewed literature. We will first highlight and explain the two contrasting understandings (corporate supervision with the focus on control and employee self-organization with a focus on freedom) and then elaborate on a comprising perspective (corporate guidance with the focus on a balanced moderation). The selected studies might not only be the domain of one approach with the complete exclusion the other approaches (shown with a " $(\mathrm{x})$ " in Table 3). This means, that the authors were very differentiated in their implications. Although, often 
the narrative put one approach in the foreground, we could still gain useful insights about another approach. Consequently, one article might be assigned to two approaches and if one approach is clearly favored " $\mathrm{x}$ " is used as an indicator.

\subsection{Corporate supervision}

To start with the corporate supervision category as displayed in Table 1 four articles $([2,32,50,68])$ were giving implications for an active and strong corporate engagement to facilitate the use. Three more articles $([5,14,31])$ were partly addressing a rigorous corporate moderation. The necessity to make the new communication environment known, safe and setting the expectation of use for the employees is one of the main reasons for a high degree of corporate engagement $[2,50,68]$. The literature based study of [68] applied the fit-viability model for accessing concerns for the public SNS and ESN use, suggesting for risk mitigation issues "corporations should establish a governance structure and policies for anticipated uses" [68; p. 215]. Further, they advocate being vocal about consequences of non-compliance. Strict display of e.g. use cases to diminish associated barriers of an unknown application scope, is also addressed in the case study of [2]. In accordance with the mentioned policy suggestion the authors formulate access control as well as monitoring and filtering recommendation. Through the latter two mechanisms the policies become enforceable and the employee usage can be analyzed. [32] argue in their research paper that functional corporate tracking and display of UGC contributors activity can "incentivize people to engage in this practice" [32; p. 13]. They even propose to use such user contribution and reputation metrics for annual employee performance reviews. This implies that employees' careers might depend on their ESN contribution profile, which bears a huge amount of organizational pressure. This need for organizational pressure is also recognized in the case study of [50] for accepting the ESN services as an "required tool at the workplace"[50; p. 11]. But their case study also reveals the need for corporate moderated UGC quality controls and access management. This relates to the access control thought of [68]. They suggest that with the help of access rights corporate authorities are not only able to predefine the nature of content but also the reach and size of audience for UGC [68]. This on the one hand is reducing the openness constraint for contributors but on the other hand demands new corporate roles like ESN managers (e.g. community or wiki manager) and supervision committees as well as top management usage display $[2,5,14,31,50,68]$. The high degree of corporate engagement is envisioned to build an organizational 'support structure' to empower and motivate UGC providers [2]. Here [68] raise the concern that some users might not be happy with direct interference, but opting the ESN UGC quality level over the single user's freedom.

Summing up (see Table 4), the active supervision is encouraging the ESN users to actively contribute because the accessibility is predicable, the use is promoted or incentivized and employees are informed about the expected use as well as the content reviews to assure quality.

Table 4 - Summary of supervision approach

\begin{tabular}{|rc|}
\hline \multicolumn{2}{|c|}{ Corporate supervision } \\
\hline Org. support structure & {$[2,5,14,31,68]$} \\
Policy/User compliance & {$[2,68]$} \\
Access control & {$[50,68]$} \\
Monitoring/Filtering & {$[2,32,68]$} \\
Organizational pressure & {$[2,32,50,68]$} \\
Content quality control & {$[2,32,50,68]$} \\
\hline
\end{tabular}

\subsection{Employee self-organization}

The second approach is motivated by the bottomup ideology of the web 2.0 roots [64] and advocates a loose moderation to comply with the user-centric ESN nature $[4,15,31,39,45,57,63,64]$. Table 1 at the beginning of the paragraph highlights that only three articles $([4,15,39])$ of the sample were in clear favor of such an employee self-organization. However, six further articles $([31,45,55,57,63,64])$ are positively discussing it with the respect to other approaches. In the literature based review of web 2.0 technologies in organizations [64] conclude that prior "highly standardised information infrastructures tightly administered by top management" [64; p. 6] stay contrasting to the new technology, especially naming the intranet as an artifact. The author reflects also on top managers' engagement but describes them as promoting key users and UGC contributors themselves, rather than UGC supervising and controlling gate keepers. Bearing in mind the power relation context, this is putting the executives on eye level with other UGC creators in the ESN [55,64]. In terms of knowledge management [39] pick up the dissimilarity with traditional centralized structures. The author reasons that the UGC is the accountability of the employee who created it and leaves only the option to let it be "governed by the employees themselves, including the choice of whom they want to distribute the self-generated content" [39; p. 162] to. However, in that literature based research agenda they acknowledge that management might need new responses to deal with unpleasant UGC. Joining in, 
[15] reflected on their mixed method case study regarding wiki use for research and development project management, that the content creator should be in charge of the reach, to increase the interaction of UGC contributors. Although that the authors of [12] remark, that the activity meta data (e.g. user A comment on user B's blog) can and should be distributed openly to give a sense of progress. This shows that the accountability of UGC lies with the creator and so does the right of administration of the sharing process. [4] identified in their study about benefits and barriers of ESN in large scale organizations, that a strong corporate engagement e.g. through policing the use, might result in dismissal by the content creators. The perceived "restrictions to freedom of expression" [4; p. 83] by users is strongly related to the willingness to contribute. Hence the high degree of user freedom und low corporate engagement is seen as a positive factor on UGC creation. Although [4] reflect that the degree of freedom is not always a choice of the company due to industry regulations and public law demands. Being more differentiated on the policy part [63] suggest that the new technology enabled collaboration is governed "on principles rather than rules" [51, p. 251.] which is in accordance with the widely mentioned permissive corporate culture $[4,15,31,57,64]$. In their focus group research with senior IT managers, they further bring up a user self-policing mechanism through the elimination of anonymity. Which means that in a professional context the vandalism [15] or extra negativity related to UGC will be governed by ESN users themselves, if those sources are also transparent and are held accountable for such doing. They do not name that mechanism directly but it relates to the later made connection of [5] and [31] to the stewardship concept. Here both suggest as evidence from their case studies that with the growing participation and active contribution of UGC the users become stewards of the ESN without a clear corporate demand and taking over new responsibility roles by themselves. By taking over a more collective understanding of the ESN benefit, the users might voluntary execute bottom-up control functions (e.g. like wiki-gardeners [31]) and consequently follow a more intrinsic pro organizational behavior.

Table 5 - Summary of self-organization

\begin{tabular}{|cc|}
\hline \multicolumn{2}{|c|}{ Employee self-organization } \\
\hline Equality & {$[55,64]$} \\
Accountability & {$[15,31,39,63]$} \\
User freedom & {$[4,15,63,64]$} \\
Corporate culture & {$[4,15,31,57,64]$} \\
Transparency & {$[4,15,63]$} \\
Stewardship & {$[31,63]$} \\
\hline
\end{tabular}

\subsection{Corporate guidance}

As the latter two approaches were quite diametric the third approach is in the middle combining both sides with a moderate corporate engagement. This approach is identified in seventeen articles (see Table 1) of the sample with five more articles not clearly advocating solely for the approach. In this moderation style the focus is on leveraging the decentralized bottom-up roots with a corporate guidance to facilitate the willingness to contribute UGC in the ESN. This tradeoff between the other approaches is underlying the ESN discussion early on and is mentioned by [23] in an examination of the enterprise knowledge management perspective and the representation of knowledge within the new web 2.0 technologies. Further [23] concluded that organizations need a plan and that the context of the use plays a role in whether a top-down or bottom-up moderation is applicable. This relates to the communicational ambidexterity mentioned first by [5] and then further elaborated in detail by [31]. In the case study of [31], the authors examined the interplay between internal organizational published content and UGC management. They evaluated the different modes of communication and applied the concept of ambidexterity to explain how these two modes can be managed side by side with the growing multi-vocal UGC from ESN usage. The resulting framework suggests enabling mechanisms - consisting of flexible governance, a set-up of communication environment and a dynamic communication culture - will nurture the communicational ambidexterity capability of corporations. This will result in a complimentary outcome of the two modes and will reflect back on the enabling mechanisms. Although [31] focus on the interplay of two different forms of internal communication they show a context depending corporate guiding hand. What can be derived from their case study is, that corporations need to be aware that their engagement is not "overpowering UGC communication" [31; p. 60]. Context is also seen as an important guideline for using a certain tool for a specific task [7]. [34] give some additional implication. They described the need for "careful" policing in combination with ESN user education in order to foster persistent UGC. This policing and guideline theme is visible in other articles $[17,28,33,38,53,63,64,70]$ but is different from the corporate supervision perspective with the education theme being in the focus, rather than the compliance character. This user education is primary associated with a corporate engagement in communicating, training and show casing the wanted ESN use, with top-management taking the active ESN user role 
model in $[13,26,28,34,70]$. Top-management commitment to ESN tools is often seen as a jump starter at the beginning [38], quality assurance $[12,14,21]$ and motivator [14,21] for UGC. Another corporate moderation is suggested by [54] in their mixed method case study examination to determine diversity among different ESN communities. They promote the idea of a templating service which guides the ESN user to the wanted UGC outcome via a corporate service orientation, but preserves enough flexibility to customize the user's own perception. The flexibility is again highlighting the before mentioned tradeoff. Here [28; p. 4075] deliver additional remark with their mixed method case study that the degree of predefined uses (here understood as the degree of corporate engagement) needs to leave "still enough freedom" for the ESN users in order to have them engaged. Further, implications are given in terms of recommendation systems to steer the users' content consumption $[54,62]$ or with a reward system and ESN management team $[12,70]$. The latter two were also part of the findings in the corporate supervision approach, but here the focus was on the UGC promotion $[12,14]$ rather than on compliance. Underlying most of the articles in this section, organizational culture $[5,12,13,14,17,31,57]$ is one of the facilitators of this moderate engagement approach, with an open and supportive culture encouraging UGC creation. Yet again the stewardship idea is visible, with corporate culture, principles and user education building the common ground for the UGC community to start an efficient self-regulation process $[13,31]$.

This said the guidance approach is touching in some means the other presented moderation approaches. But with the difference that the governance mechanisms are suggesting to have an adjustable structure, the architecture of the ESN tools is defined but open to context depending customization, a service orientation in ESN management and a voluntary user education. This will lead to principle based moderation which gives the UGC provider enough free space and adequate support structure to encourage the content creation willingness.

Table 6 - Summary of guidance approach

\begin{tabular}{|rc|}
\hline \multicolumn{2}{|c|}{ Corporate guidance } \\
\hline Context & {$[17,28,31,33,34,38,53,55,63,64,70]$} \\
Dynamic governance & {$[7,23,31,57]$} \\
User education & {$[17,28,33,38,53,63,64,70]$} \\
Top-management & {$[12,13,14,21,26,28,34,70]$} \\
commitment & {$[28,54]$} \\
Service orientation & {$[13,31]$} \\
Stewardship & {$[5,12,13,14,17,31,57]$} \\
Corporate culture & \\
\hline
\end{tabular}

\section{Discussion, implications and recommendations}

With the classification of methods and concepts under each of the three generic approaches, it became evident that all sources advocate that they will lead to a high degree in UGC content. If so, it would be irrelevant for corporations to choose a moderation form. We, however, will now review the findings above with the help of some critical success factors for ESN adoption and web 2.0 phenomena. The outcome will be a classification of the three approaches in terms of the encouragement of UGC and the prior described degree of corporate engagement. We start the discussion with addressing the critical mass encouragement which is believed to deliver the selfsustaining factor for an ESN next to other computer mediated communication and collaboration mediums $[12,17,27,29,34]$. The main argument for the supervision approach is the generation of a clearly structured and reviewed environment. A revision or even surveillance strongly influences the privacy of an ESN user. Here recent studies revealed that privacy concerns have a negative impact on the intention to use and sharing behaviors of ESN users [10,52]. Further, the reduced freedom for users to generate content is contradicting with e.g. corporate blog reader attraction, who are seeking greater UGC diversity [62]. On the other hand, incentivizing UGC, as proposed by some authors, might be helping to offset the negative impact of privacy concerns and restricted freedom. But this can be a complex task in terms of threshold setting and quality assurance when considering current results of [47] for public SNS. The discussion showed, that a strong corporate engagement has an ambivalent status, but on the apprehension of the ESN being perceived as a topdown corporate communication channel (e.g. [17]) we propose that the corporate supervision approach will lead to a relatively low degree of UGC.

The self-regulation approach is taking the freedom and variety argument for its sakes and proclaims to generate a high level of UGC. However, when looking at this approach a mandatory pre-condition is an completely open communication culture without hierarchy constraints $[5,17]$. Otherwise the content generation might be harmed because of its professional environment, where the UGC creators' reputation is at stake and is conflicting with a nonguided trial and error use approach [23]. Also relating to this the accountability and audience structure empowerment is identified to create "a sense of anxiety" [66; p. 18] for ESN users. The content creator needs to think about with whom he wants to share and might be confronted with leaving people outside of the 
information loop. The proclaimed freedom bears a lot more user responsibility through active partaking in power relation setting. E.g. the feeling of 'anxiety' can further escalate when the question of distribution was prior placed with the employees' supervisor, or the latter needs to intervene if a discussion takes a wrong way due to missing cues [58]. Another UGC distribution barrier can originate from professional users more self-oriented and calculated profile in their contributing and sharing behavior [52]. Moreover, the self-orientation might make it difficult to build the proclaimed common basis which eventually leads to the stewardship phenomenon. Hence, without setting common and accepted principles, this state might not be achieved. This deems the public use inspired bottom-up moderation as not sufficient enough to guarantee a high UGC share. Considering the investments corporations made for such software applications it seems risky to just let them develop their way next to other traditional and largely accepted communication and collaboration technologies (like e.g. email). Comparing the self-organization approach, it is rather encouraging UGC than the supervision style, in the right organizational setting.

Moving on to the third approach it is clear that the moderate positioning bears a huge advantage. The option to combine the wanted benefits and diminish the undesired accompanying effects of both other approaches sounds desirable. This approach can be used to nurture the preferred organizational setting and to embrace an open communication culture through educating and guiding the UGC provider. This educated user will, on the one hand, have the described safe and known environment and on the other hand will find enough space but also support/service to express themselves. Nevertheless, this approach is a difficult task for the organization, as they have to provide service without giving the impression of branding the employee identity, which brings us also to the capability of ambidexterity of [31]. Even further the moderation will need to be reconfiguring context depended, but has to provide enough stability to not create uncertainty again, which might harm UGC creation. This might help to build the base for "involvement and trust" [16; p. 43] and a culture "based on collectivism and low power distance" [16; p. 43] to generate managerial setting for a stewardship relation. With the idea of educating the users and providing them a service oriented structure the degree of UGC is assessed higher than for the selforganization approach. A summary of the assessment from degree of corporate engagement and the added encouragement level of UGC can be found in Figure1.

From this discussion, we can not only derive the classification but also the need for moderation of an
ESN at a certain time. Even in the low corporate engagement approach, top-management engaging as users themselves is somehow an act of passive moderation with the desire to motivate the users to generate content in the ESN. Also the prior mentioned investment argument will ask for a justification plan prior to the investment (e.g. [24]) or bring a post implementation review with it (e.g. [25]; even though sufficient ex-post reviews are not that prevalent). As the communication in the ESN evolves over time (e.g. [21]) the need for a certain corporate engagement is most certainly variating over time and maturation phases as well (e.g. active top management involvement as jump start in the beginning). Consequently, corporations will probably need to constantly reassess their engagement level based on the ESN usage evolution.

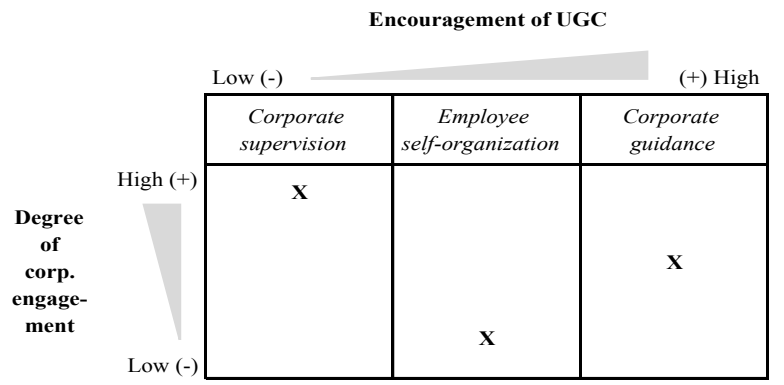

Figure 1 - Assessment of approaches

\section{Limitations and outlook}

Despite using a systematic approach there might be some limitations to this paper. First of all, we included only conference and journal papers except of [67]. This was done as a quality assurance, to have only fore mostly peer reviewed sources. Although, the main base of the articles are quality reviewed publications some smaller conference proceedings were included due to the subject relevancy. To account for the often made critiques of an Anglo-American research perspective, we included the EJIS and ECIS proceedings. Further, the initial keyword search was predefined in a broader sense, covering ESN literature in general. A few publications might have been neglected in the processes, which would have been covered, with an inductive second keyword search.

As this literature review aimed to identify implication given for practitioners, we compared findings of different methodologies and unique case study results. As this topic is quite young there was no other way as to review those publications together. Furthermore, not every article was solely examining the moderation question as the main research subject. 
Nevertheless, this question was often a byproduct of the discussion and practical implications.

As we tried to answer the RQ from a literature base, we might lack the validation of own collected empirical data. Further, it became evident that those moderation approaches might depend on the context of maturation stages of the ESN implementation (e.g. [30] tracking the ESN activity at the start). Hence, it should be investigated if one approach might be more suitable in a certain stage. Here we propose a longitude case study, examining the evolution of the corporate engagement level in different ESN maturation stages. Furthermore, we did not consider cultural differences, which might influence the perception of a certain moderation approach and impact the users encouragement for UGC [61]. Here a survey based examination in a multinational enterprise with an ESN system might provide deeper insights of the espoused national culture impact on the user content generation.

\section{Conclusions}

Summarizing the research findings, we firstly identified three different corporate ESN moderation styles: corporate supervision, employee selforganization and corporate guidance. The corporate supervision approach tries to overcome the unstructured nature of social technology in a corporate context with strict policing, content monitoring and compliance characteristics. Contrary to this high corporate engagement style we presented the diametric employee self-organization approach. This moderation style is taking the transparency induced accountably of ESN services as a self-regulating mechanism. Although, the degree of corporate engagement is much lower there is still a passive engagement resulting from active top management usage. Then we presented the in the literature by far leading corporate guidance approach. This approach is located in between the latter two diametric styles. Here the corporation is educating the users about the ESN properties and possibilities to change the current work practices. The degree of corporate engagement is higher as in the self-organization style, but still lower compared to the corporate supervision approach.

Secondly, we discussed which of the approaches is encouraging more UGC in order to foster ESN activity. We credited the corporate guidance approach with being an UGC motivator followed by the selforganization approach trailed by the corporate supervision style.

Our third finding was the need for a corporate moderation, based on the competition with other communication technologies and the monetary IT investment argument.
With comparing diverse topics and searching for moderation methods identified in the ESN literature, we could build a first generalization of the three named approaches. This added novelty to the ongoing discourse of how to establish an ESN within an enterprise and to attract UGC. Building on this literature review we provide a frame for future discussions. For practitioners we highlight, that the moderation level is related to the users' contribution behavior and that they have to think about the consequences of a chosen corporate engagement level.

\section{References}

[1] Ali-Hassan, H., Nevo, D., and Wade, M. Linking Dimensions of Social Media Use to Job Performance: The Role of Social Capital. The Journal of Strategic Information Systems 24, 2 (2015), pp. 65-89.

[2] Annabi, H., McGann, S.T., Pels, S., Arnold, P., and Rivinus, C. Guidelines to Align Communities of Practice with Business Objectives: An Application of Social Media. Proc. of the Hawaii International Conference on System Sciences (HICSS), (2012), pp. 3869-3878.

[3] Aral, S., Dellarocas, C., and Godes, D. Introduction to the Special Issue - Social Media and Business Transformation: A Framework for Research. Information Systems Research 24, 1 (2013), pp. 3-13.

[4] Baltatzis, G., Ormrod, D.G., and Grainger, N. Social Networking Tools for Internal Communication in Large Organizations: Benefits and Barriers. Proc. of the Australasian Conference on Information Systems (ACIS), (2008), pp. 76-86.

[5] Baptista, J. and Galliers, R.D. Social Media as a Driver for New Rhetorical Practices in Organisations. Proc. of the Hawaii International Conference on System Sciences (HICSS), (2012), pp. 3540-3549.

[6] Behrendt, S., Klier, M., Klier, J., Richter, A., and Wiesneth, K. The Impact of Formal Hierarchies on Enterprise Social Networking Behavior. Proc. of the International Conference on Information Systems (ICIS), (2015), pp. 1-19.

[7] Boateng, R., Malik, A., and Mbarika, V. Web 2.0 and organizational learning: Conceptualizing the link. Proc. of the American Conference on Information Systems (AMCIS), (2009), pp. 1-12.

[8] boyd, danah m. and Ellison, N.B. Social Network Sites: Definition, History, and Scholarship. Journal of ComputerMediated Communication 13, 1 (2007), pp. 210-230.

[9] Brocke, J. vom, Simons, A., Niehaves, B., Reimer, K., Plattfaut, R., and Cleven, A. Reconstructing the Giant: On the Importance of Rigour in Documenting the Literature. Proc. of the European Conference on Information Systems (ECIS), (2009), pp. 1-16.

[10] Buettner, R. Analyzing the Problem of Employee Internal Social Network Site Avoidance: Are Users Resistant due to Their Privacy Concerns? Proc. of the Hawaii International Conference on System Sciences (HICSS), (2015), pp. 1819-1828.

[11] Chen, W. and Hirschheim, R. A paradigmatic and 
methodological examination of information systems research from 1991 to 2001. Information Systems Journal 14, 3 (2004), pp. 197-235.

[12] Chin, C.P.-Y., Evans, N., and Choo, K.-K.R. Exploring Factors Influencing the Use of Enterprise Social Networks in Multinational Professional Service Firms. Journal of Organizational Computing and Electronic Commerce 25, 3 (2015), pp. 289-315.

[13] Chin, C.P.-Y., Evans, N., Choo, R.K., and Tan, F.B. What Influences Employees to Use Enterprise Social Networks? A Socio-Technical Perspective. PACIS 2015 Proc., (2015).

[14] Chin, C.P. and Choo, K.R. Enterprise Social Networks : A Successful Implementation within a Telecommunication Company Full Paper. Proc. of the American Conference on Information Systems (AMCIS), (2015), pp. 1-11.

[15] Danis, C. and Singer, D. A Wiki Instance in the Enterprise. Proc. of the Conference on Computer supported cooperative work - (CSCW), ACM Press (2008), pp. 495504.

[16] Davis, J.H., Schoorman, F.D., and Donaldson, L. Toward a Stewardship Theory of Management. Academy of Management Review 22, 1 (1997), pp. 20-47.

[17] Denyer, D., Parry, E., and Flowers, P. "Social", "Open" and "Participative"? Exploring Personal Experiences and Organisational Effects of Enterprise2.0 Use. Long Range Planning 44, 5-6 (2011), pp. 375-396.

[18] Elliot, S. Transdisciplinary Perspectives on Environmental Sustainability: A Resource Base and Framework for It-Enabled Business Transformation. MIS Quarterly 35, 1 (2011), pp. 197-236.

[19] Ellison, N.B., Gibbs, J.L., and Weber, M.S. The Use of Enterprise Social Network Sites for Knowledge Sharing in Distributed Organizations: The Role of Organizational Affordances. American Behavioral Scientist 59, 1 (2015), pp. 103-123.

[20] Gaß, O., Ortbach, K., Kretzer, M., Maedche, A., and Niehaves, B. Conceptualizing Individualization in Information Systems - A Literature Review. Communications of the Association for Information Systems 37, (2015), pp. 64-88.

[21] Gibbs, J.L., Eisenberg, J., Rozaidi, N.A., and Gryaznova, A. The "Megapozitiv" Role of Enterprise Social Media in Enabling Cross-Boundary Communication in a Distributed Russian Organization. American Behavioral Scientist 59, 1 (2015), pp. 75-102.

[22] Gray, P.H., Parise, S., and Iyer, B. Innovation Impacts of Using Social Bookmarking Systems. MIS Quarterly 35, 3 (2011), pp. 629-643.

[23] Grudin, J. Enterprise Knowledge Management and Emerging Technologies. Proc. of the Hawaii International Conference on System Sciences (HICSS), (2006), pp. 1-10.

[24] Gunasekaran, A., Ngai, E.W.T., and McGaughey, R.E. Information Technology and Systems Justification: A Review for Research and Applications. European Journal of Operational Research 173, 3 (2006), pp. 957-983.

[25] Gwillim, D., Dovey, K., and Wieder, B. The Politics of Post-Implementation Reviews. Information Systems Journal 15, 4 (2005), pp. 307-319.

[26] Hamadani Janes, S., Patrick, K., and Dotsika, F. Implementing a Social Intranet in a Professional Services
Environment through Web 2.0 Technologies. The Learning Organization 21, 1 (2014), pp. 26-47.

[27] Harden, G. Knowledge Sharing in the Workplace: A Social Networking Site Assessment. Proc. of the Hawaii International Conference on System Sciences (HICSS), (2012), pp. 3888-3897.

[28] Herzog, C. and Richter, A. Use Cases as a Means to Support the Appropriation of Enterprise Social Software. Proc. of the Hawaii International Conference on System Sciences (HICSS), (2016), pp. 4072-4081.

[29] Herzog, C., Richter, A., and Steinhüser, M. Towards a Framework for the Evaluation Design of Enterprise Social Software. Proc. of the International Conference on Information Systems (ICIS), (2015), pp. 1-20.

[30] Herzog, C., Richter, A., Steinhüser, M., Hoppe, U., and Koch, M. Methods and Metrics for Measuring the Success of Enterprise Social Software - What We can Learn from Practice and Vice Versa. Proc. of the European Conference on Information Systems (ECIS), (2013), pp. 1-12.

[31] Huang, J., Baptista, J., and Newell, S. Communicational Ambidexterity as a New Capability to Manage Social Media Communication within Organizations. The Journal of Strategic Information Systems 24, 2 (2015), pp. 49-64.

[32] Huang, Y., Singh, P., and Ghose, A. Show me the Incentives: A Dynamic Structural Model of Employee Blogging Behavior. Proc. of the International Conference on Information Systems (ICIS), (2010), pp. 1-15.

[33] Husin, M. and Hanisch, J. Social Media and Organisation Policy ( Someop ): Finding the perfect Balance. Proc. of the European Conference on Information Systems (ECIS), (2011), pp. 1-12.

[34] Jackson, A., Yates, J., and Orlikowski, W. Corporate Blogging: Building community through persistent digital talk. Proc. of the Hawaii International Conference on System Sciences (HICSS), (2007), pp. 80-80.

[35] Kane, G., Alavi, M., Labianca, G., and Borgatti, S. What's Different about Social Media Networks? A Framework and Research Agenda. MIS Quarterly 38, 1 (2014), pp. 275-304.

[36] Kaplan, A.M. and Haenlein, M. Users of the World, Unite! The Challenges and Opportunities of Social Media. Business Horizons 53, 1 (2010), pp. 59-68.

[37] Karoui, M., Dudezert, A., and Leidner, D.E. Strategies and Symbolism in the Adoption of Organizational Social Networking Systems. The Journal of Strategic Information Systems 24, 1 (2015), pp. 15-32.

[38] Kolari, P., Finin, T., abd Y. Yesha, K.L., Yesha, Y., Perelgut, S., and Hawkins, J. On the Structure, Properties and Utility of Internal Corporate Blogs. In Proc. of the International Conference on Weblogs and Social Media (ICWSM), (2007).

[39] von Krogh, G. How does Social Software Change Knowledge Management? Toward a Strategic Research Agenda. The Journal of Strategic Information Systems 21, 2 (2012), pp. 154-164.

[40] von Krogh, G., Rossi-Lamastra, C., and Haefliger, S. Phenomenon-based Research in Management and Organisation Science: When is it Rigorous and Does it Matter? Long Range Planning 45, 4 (2012), pp. 277-298.

[41] Kuegler, M., Smolnik, S., and Kane, G. What's in IT for Employees? Understanding the Relationship Between 
Use and Performance in Enterprise Social Software. The Journal of Strategic Information Systems 24, 2 (2015), pp. 90-112.

[42] Kügler, M. and Smolnik, S. Just for the Fun of It? Towards a Model for Assessing the Individual Benefits of Employees' Enterprise Social Software Usage. Proc. of the Hawaii International Conference on System Sciences (HICSS), (2013), pp. 3614-3623.

[43] Leonardi, P.M. Social Media, Knowledge Sharing, and Innovation: Toward a Theory of Communication Visibility. Information Systems Research 25, 4 (2014), pp. 796-816.

[44] Leonardi, P.M. Ambient Awareness and Knowledge Acquisition: Using Social Media to Learn "Who Knows What" and "Who Knows Whom. MIS Quarterly 39, 4 (2015), pp. 747-762.

[45] Leonardi, P.M., Huysman, M., and Steinfield, C. Enterprise Social Media: Definition, History, and Prospects for the Study of Social Technologies in Organizations. Journal of Computer-Mediated Communication 19, 1 (2013), pp. 1-19.

[46] Levy, Y. and Ellis, T.J. A systems approach to conduct an effective literature review in support of information systems research. Informing Science Journal 9, (2006), pp. 181-212.

[47] Liu, Y. and Feng, J. Can Monetary Incentives Increase UGC Contribution? The Motivation and Competition Crowding Out. Proc. of the International Conference on Information Systems (ICIS), (2015), pp. 1-16.

[48] Loebbecke, C. and Leidner, D. The Contribution of Top IS Publications to Subsequent Research: A Citation Analysis. Communications of the Association for Information Systems 30, (2012), pp. 423-438.

[49] Lowry, P.B., Romans, D., and Curtis, A. Global Journal Prestige and Supporting Disciplines : A Scientometric Study of Information Systems Journals. Journal of the Association for Information Systems 5, 2 (2004), pp. 29-77.

[50] Mansour, O., Abusalah, M., and Askenäs, L. Wiki Collaboration in Organizations: An Explorartory Study. Proc. of the European Conference on Information Systems (ECIS), (2011), pp. 1-14.

[51] McAfee, A.P. Enterprise 2.0: The Dawn of Emergent Collaboration. MIT Sloan Management Review 47, 3 (2006), pp. 21-28.

[52] Mettler, T. and Winter, R. Are Business Users Social? A Design Experiment Exploring Information Sharing in Enterprise Social Systems. Journal of Information Technology, (2015), pp. 1-14.

[53] Miles, S.J. and Mangold, W.G. Employee Voice: Untapped Resource or Social Media Time Bomb? Business Horizons 57, 3 (2014), pp. 401-411.

[54] Muller, M., Ehrlich, K., Matthews, T., Perer, A., Ronen, I., and Guy, I. Diversity Among Enterprise Online Communities. Proc. of the SIGCHI Conference on Human Factors in Computing Systems (CHI), (2012), pp. 28152824.

[55] Osch, W. van, Steinfield, C.W., and Balogh, B.A. Enterprise Social Media: Challenges and Opportunities for Organizational Communication and Collaboration. Proc. of the Hawaii International Conference on System Sciences (HICSS), (2015), pp. 763-772.

[56] Peffers, K. and Tang, Y. Identifying and Evaluating the
Universe of Outlets for Information Systems Research: Ranking The Journals. The Journal of Information Technology Theory and Application 5, 1 (2003), pp. 63-84. [57] Razmerita, L., Kirchner, K., and Nabeth, T. Social Media in Organizations: Leveraging Personal and Collective Knowledge Processes. Journal of Organizational Computing and Electronic Commerce 24, 1 (2014), pp. 7493.

[58] Richter, A., Hetmank, C., Klier, J., Klier, M., and Muller, M. Enterprise Social Networks from a Manager's Perspective. Proc. of the Hawaii International Conference on System Sciences (HICSS), (2016), pp. 4242-4251.

[59] Richter, D., Riemer, K., and vom Brocke, J. Internet Social Networking. Business \& Information Systems Engineering 3, 2 (2011), pp. 89-101.

[60] Riemer, K., Finke, J., and Hovorka, D. Bridging or Bonding: Do Individuals gain Social Capital from Participation in Enterprise Social Networks? Proc. of the International Conference on Information Systems (ICIS), (2015), pp. 1-20.

[61] Schlagwein, D. and Prasarnphanich, P. Cultural Determinants of Organizational Social Media Adoption. Proc. of the European Conference on Information Systems (ECIS), (2011), pp. 1-11.

[62] Singh, P.V., Sahoo, N., and Mukhopadhyay, T. How to Attract and Retain Readers in Enterprise Blogging? Information Systems Research 25, 1 (2014), pp. 35-52.

[63] Smith, H. a. and McKeen, J.D. Enabling collaboration with IT. Communications of the Association for Information Systems 28, 1 (2011), pp. 243-254.

[64] Stenmark, D. Web 2 . 0 in the Business Environment : The New Intranet or a Passing Hype ? Proc. of the European Conference on Information Systems (ECIS), (2008), pp. 112.

[65] Te'eni, D., Rowe, F., Ågerfalk, P.J., and Lee, J.S. Publishing and getting published in EJIS: marshaling contributions for a diversity of genres. European Journal of Information Systems 24, 6 (2015), pp. 559-568.

[66] Treem, J.W. Social Media as Technologies of Accountability: Explaining Resistance to Implementation Within Organizations. American Behavioral Scientist 59, 1 (2014), pp. 53-74.

[67] Treem, J.W. and Leonardi, P.M. Social Media Use in Organizations: Exploring the Affordances of Visibility, Editablity, Persistence, and Association. Communication Yearbook 36, (2012), pp. 143-189.

[68] Turban, E., Bolloju, N., and Liang, T.-P. Enterprise Social Networking: Opportunities, Adoption, and Risk Mitigation. Journal of Organizational Computing and Electronic Commerce 21, 3 (2011), pp. 202-220.

[69] Urbach, N., Morana, S., and Maedche, A. Are you a Maverick? Towards a Segmentation of Collaboration Technology Users. Proc. of the International Conference on Information Systems (ICIS), (2015), pp. 1-11.

[70] Wang, T., Jung, C.-H., Kang, M.-H., and Chung, Y.-S. Exploring Determinants of Adoption Intentions Towards Enterprise 2.0 Applications: An Empirical Study. Behaviour \& Information Technology 33, 10 (2014), pp. 1048-1064.

[71] Webster, J. and Watson, R. Analyzing the Past to Prepare for the Future: Writing a Literature Review. MIS Quarterly 26, 2 (2002), pp. 13-23. 